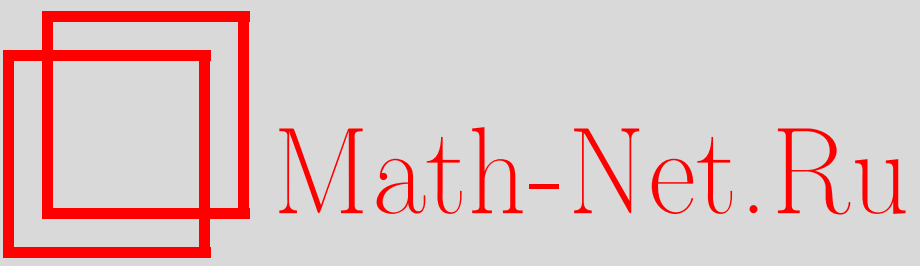

П. А. Крутицкий, О фредгольмовости систем интегральных уравнений, $У M H$, 1998, том 53, выпуск 6, 253-254

DOI: https://doi.org/10.4213/rm100

Использование Общероссийского математического портала Math-Net.Ru подразумевает, что вы прочитали и согласны с пользовательским соглашением

http://www.mathnet.ru/rus/agreement

Параметры загрузки:

IP: 34.239 .49 .27

26 апреля 2023 г., 14:09:52 


\section{О ФРЕДГОЛЬМОВОСТИ СИСТЕМ ИНТЕГРАЛЬНЫХ УРАВНЕНИЙ}

\section{П. А. Крутицкий}

На координатной оси $O s$ введем две совокупности отрезков $\Gamma=\bigcup_{n=1}^{N} \Gamma_{n}$ и $\gamma=\bigcup_{m=1}^{M} \gamma_{m}$, где $\Gamma_{n}=\left[a_{n}, b_{n}\right], \gamma_{m}=\left[\alpha_{m}, \beta_{m}\right], N, M \geqslant 0$. При этом все отрезки не имеют общих точек, за тем исключением, что концы $\Gamma$ могут совпадать с концами $\gamma$. Положим $D=\Gamma \cup \gamma$. Рассмотрим систему интегральных уравнений на $D$ относительно функции $\mu(s)$ :

$$
\begin{gathered}
\frac{1}{\pi} \int_{\Gamma} \frac{\mu(\sigma)}{\sigma-s} d \sigma+\int_{D} \mu(\sigma) v(s, \sigma) d \sigma=f(s), \quad s \in \Gamma, \\
\mu(s)+\int_{D} \mu(\sigma) w(s, \sigma) d \sigma=f(s), \quad s \in \gamma, \\
\int_{D} \mu(\sigma) U_{n}(\sigma) d \sigma=\varphi_{n}, \quad n=1, \ldots, N .
\end{gathered}
$$

Здесь $v(s, \sigma), w(s, \sigma), U_{1}(s), \ldots, U_{N}(s), f(s)$ - заданные функции, а $\varphi_{1}, \ldots, \varphi_{N}$ - заданные константы. Относительно заданных функций введем следующие предположения, которые в дальнейшем будем называть условиями $\mathbf{K}: w(s, \sigma) \in C^{0}(\gamma \times D)$, либо $w(s, \sigma)$ - полярное ядро на $\gamma \times D$; $v(s, \sigma) \in C^{0, \lambda}(\Gamma \times D) ; f(s) \in C^{0, \lambda}(\Gamma) \oplus C^{0}(\gamma)$ с $\lambda \in(0,1]$; функции $U_{1}(s), \ldots, U_{N}(s)$ непрерывны на $\gamma$ и во внутренних точках $\Gamma$, а на концах $\Gamma$ могут иметь логарифмические особенности.

Для простоты показатель Гёльдера для $v(s, \sigma)$ и $f(s)$ считается одинаковым. Если на практике эти показатели различны, то в качестве $\lambda$ всегда можно взять наименьший. Решение системы уравнений (1) будем разыскивать в пространстве $C_{q}^{\omega}(\Gamma) \oplus C^{0}(\gamma) \mathrm{c} \omega \in(0,1], q \in[0,1)$.

ОПРЕДЕлЕниЕ. Функция $\mu(s)$ принадлежит пространству $C_{q}^{\omega}(\Gamma)$ с $\omega \in(0,1], q \in[0,1)$, если функция $\nu(s)=\mu(s) \prod_{n=1}^{N}\left|s-a_{n}\right|^{q}\left|s-b_{n}\right|^{q}$ принадлежит пространству Гёльдера $C^{0, \omega}(\Gamma)$ с показателем $\omega$, и $\|\mu\|_{C_{q}^{\omega}(\Gamma)}=\|\nu\|_{C^{0, \omega}(\Gamma)}$.

Первьй интеграл в (1a) является сингулярньм и понимается в смысле главного значения. Тем самым, система (1) состоит из сингулярного интегрального уравнения 1-го рода (1a), уравнения 2-го рода с непрерьвным или полярным ядром (1b) и набора дополнительных условий (1c).

Лемма 1. Пусть выполнены условия К. Если на каком-либо отрезке $\gamma_{m}(m=$ $1, \ldots, M)$ выполнень условия периодичности: $f\left(\alpha_{m}\right)=f\left(\beta_{m}\right), w\left(\alpha_{m}, \sigma\right)=w\left(\beta_{m}, \sigma\right)$ при любом $\sigma \in D$, то всякое решение системы (1) также удовлетворяет условию периодичности на $\gamma_{m}: \mu\left(\alpha_{m}\right)=\mu\left(\beta_{m}\right)$.

Доказательство леммы проводится непосредственной проверкой с использованием уравнения (1b). Фредгольмовость системы (1) можно доказать сведя ее к эквивалентному уравнению Фредгольма 2-го рода в Банаховом пространстве.

ТеОРема 1. Пусть выполнень условия $\mathbf{K}$, тогда: 1) система уравнений (1) фредгольмова; 2) все решения системь (1) лежат в $C_{1 / 2}^{p}(\Gamma) \oplus C^{0}(\gamma)$ с $p=\min \{1 / 2, \lambda\}$.

СлеДСТвИЕ 1. Пусть выполнень условия К. Если однородная система (1) имеет только тривиальное решение, то неоднородная система (1) имеет единственное решение $\mu(s) \in C_{1 / 2}^{p}(\Gamma) \oplus C^{0}(\gamma)$ при любой правой части (т.е. при любой функиии $f(s)$, удовлетворяющей условиям $\mathbf{K}$ и любом наборе констант $\left.\varphi_{1}, \ldots, \varphi_{N}\right)$.

Через $\delta(s, \mathscr{L})$, где $\mathscr{L} \subset D$, будем обозначать характеристическую функцию множества $\mathscr{L}$, т.е. $\delta(s, \mathscr{L})=0$, если $s \notin \mathscr{L}$, и $\delta(s, \mathscr{L})=1$, если $s \in \mathscr{L}$. 
Пусть $\Gamma=\Gamma^{1} \cup \Gamma^{2}, \Gamma^{1}=\bigcup_{n=1}^{N_{0}} \Gamma_{n}, \Gamma^{2}=\bigcup_{n=N_{0}+1}^{N} \Gamma_{n}$. Рассмотрим систему, включающую уравнение с логарифомическим ядром:

$$
\begin{gathered}
\frac{1}{\pi} \int_{\Gamma^{1}} \frac{\mu(\sigma)}{\sigma-s} d \sigma+\int_{D} \mu(\sigma) V(s, \sigma) d \sigma=f(s), \quad s \in \Gamma^{1}, \\
-\frac{1}{\pi} \int_{\Gamma^{2}} \mu(\sigma) \ln |s-\sigma| d \sigma+\int_{D} \mu(\sigma) u(s, \sigma) d \sigma=\mathscr{F}(s), \quad s \in \Gamma^{2}, \\
\mu(s)+\int_{D} \mu(\sigma) w(s, \sigma) d \sigma=f(s), \quad s \in \gamma, \\
\int_{D} \mu(\sigma) U_{n}(\sigma) d \sigma=\varphi_{n}, \quad n=1, \ldots, N_{0} .
\end{gathered}
$$

Здесь $V(s, \sigma), u(s, \sigma), w(s, \sigma), U_{1}(s), \ldots, U_{N_{0}}(s), \mathscr{F}(s), f(s)$ - заданные функции, а $\varphi_{1}, \ldots, \varphi_{N_{0}}$ - заданные константы. Предположим, что заданные функции удовлетворяют условиям гладкости $\mathbf{K}_{1}: V(s, \sigma) \in C^{0, \lambda}\left(\Gamma^{1} \times D\right) ; u_{s}^{\prime}(s, \sigma) \in C^{0, \lambda}\left(\Gamma^{2} \times D\right) ; \mathscr{F}(s) \in C^{1, \lambda}\left(\Gamma^{2}\right) ; f(s) \in C^{0, \lambda}\left(\Gamma^{1}\right) \oplus$ $C^{0}(\gamma)$ с $\lambda \in(0,1]$; функция $w(s, \sigma)$ та же, что и в условиях $\mathbf{K}$; функции $U_{1}(s), \ldots, U_{N_{0}}(s)$ непрерывны на $\gamma$ и во внутренних точках $\Gamma$, а на концах $\Gamma$ могут иметь логарифмические особенности.

Решение системы уравнений (2) разыскивается в пространстве $C_{q}^{\omega}(\Gamma) \oplus C^{0}(\gamma)$ с $\omega \in(0,1]$, $q \in[0,1)$. Для системы (2) верна лемма 1 . Продифференцировав уравнеие $(2 \mathrm{~b})$ по $s$, заметим, что система (2) эквивалентна системе (1), где $v(s, \sigma)=\delta\left(s, \Gamma^{1}\right) V(s, \sigma)+\delta\left(s, \Gamma^{2}\right) u_{s}^{\prime}(s, \sigma)$, функция $f(s)$ на $\Gamma^{2}$ равна $\mathscr{F}^{\prime}(s)$ и для $n=N_{0}+1, \ldots, N: \varphi_{n}=\mathscr{F}\left(a_{n}\right), U_{n}(\sigma)=$ $-\pi^{-1} \delta\left(\sigma, \Gamma^{2}\right) \ln \left|a_{n}-\sigma\right|+u\left(a_{n}, \sigma\right)$. Функции $w(s, \sigma), v(s, \sigma), f(s), U_{1}(\sigma), \ldots, U_{N}(\sigma)$ в полученной системе (1) удовлетворяют условиям $\mathbf{K}$.

Теорема 2. Пусть выполнены условия $\mathbf{K}_{1}$, тогда система (2) әквивалентна системе (1) и для системы (2) справедливы все результаты, приведеннье в теореме 1 для системь (1).

СледСтвиЕ 2. Пусть выполнены условия $\mathbf{K}_{1}$. Если однородная система (2) имеет только тривиальное решение, то неоднородная система (2) имеет единственное решение $\mu(s) \in C_{1 / 2}^{p}(\Gamma) \oplus C^{0}(\gamma),(p=\min \{1 / 2, \lambda\})$ при любой правой части (т.е. при любых функииях $f(s), \mathscr{F}(s)$, удовлетворяющих условиям $\mathbf{K}_{1}$ и любом наборе констант $\left.\varphi_{1}, \ldots, \varphi_{N_{0}}\right)$.

Работа частично поддержана грантом РФФИ № 96-01-01411.

\section{СПИСОК ЛИТЕРАТУРЫ}

[1] Мусхелишвили Н.И. Сингулярные интегральные уравнения. М.: Наука, 1968. [2] Крейн С. Г. (ред. ) Функциональный анализ. М.: Наука, 1964.

Московский государственньй

Принято редколлегией университет им. М. В. Ломоносова 02.09.1998 